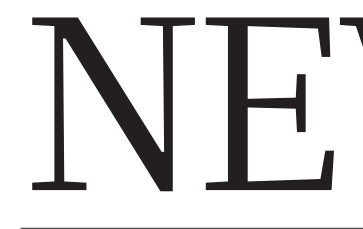

DATA-MINING Young scientists are the most open to new ideas p.283
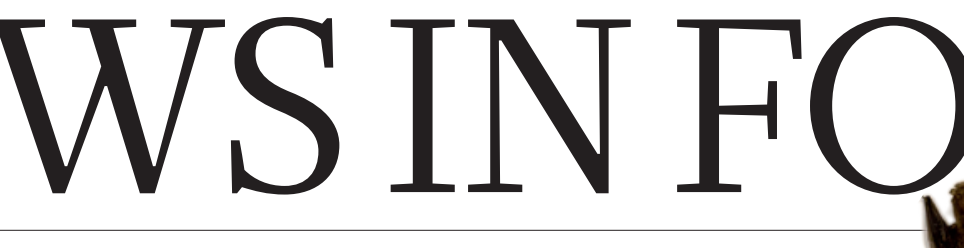

LINGUISTICS Eastern steppe

tipped as seat of Indo-

European languages p.284
BIODEFENCE Miniature

human organs grown on plastic chips $\mathbf{p . 2 8 6}$
TAXONOMY Collections grow in value even as they fall into decline $\mathbf{p . 2 9 2}$

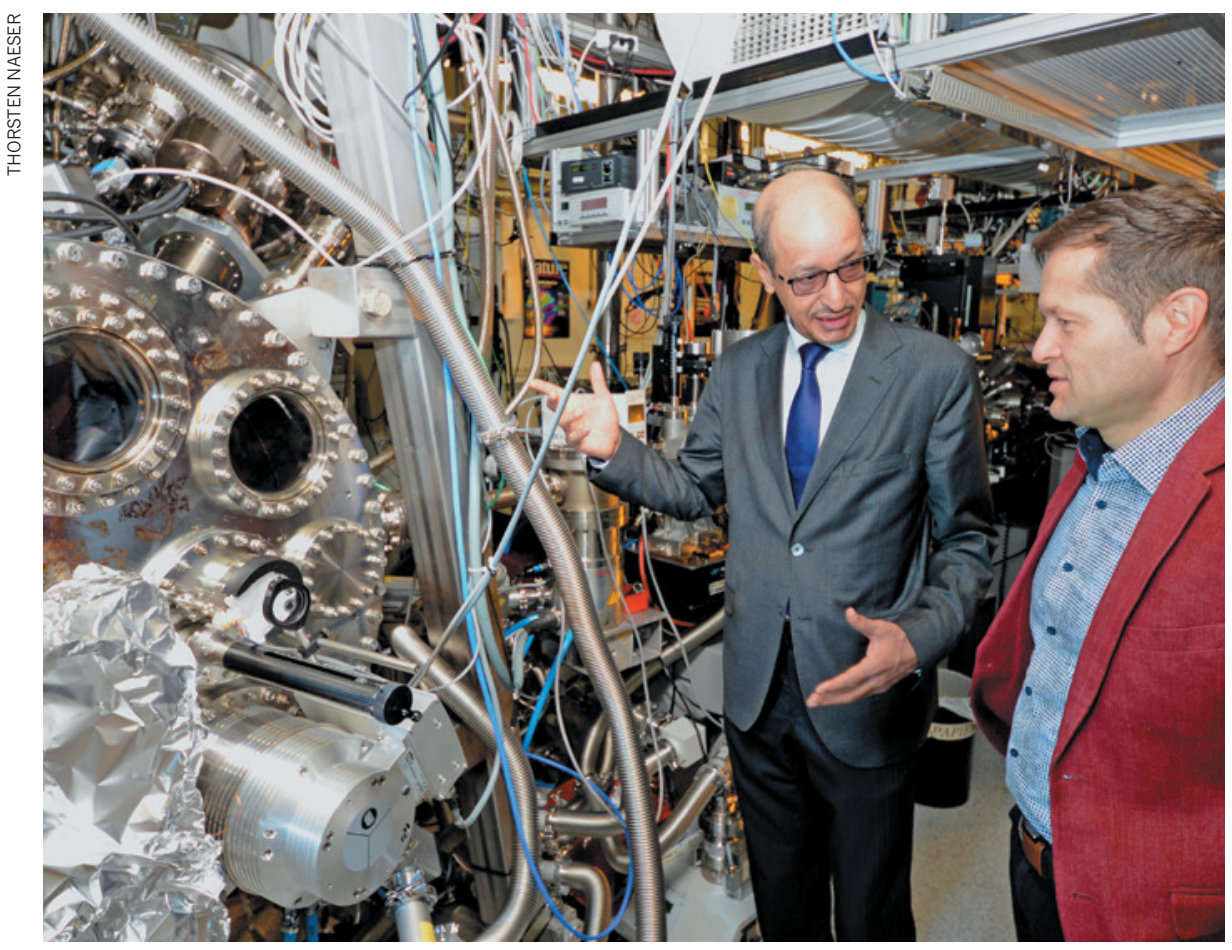

Abdallah Azzeer (left) and Ferenc Krausz head up a collaboration at King Saud University in Riyadh.

\title{
ARAB SCIENCE
}

\section{Saudi Arabia opens top-notch laser lab}

\section{Facility will study biomedical uses at attosecond resolution.}

\section{BY ALISON ABBOTT}

A cutting-edge laser facility - the first of its kind in the Arab world - opened this week at Saudi Arabia's oldest and largest university. The launch pushes forward the country's ambitions to become a leader in science and builds on a collaboration with Western scientists that has required some cultural adjustments.

The Attosecond Science Laboratory at King Saud University (KSU) in Riyadh hosts an 'attosecond laser', which generates ultrashort pulses of light, lasting just a few billionths of a billionth of a second, that can image otherwise invisible electrons as they move similarly fast within atoms. Attosecond lasers were invented in 2001, and facilities now exist at dozens of sites around the world. The Saudi Arabian facility is the result of a collaboration that began in 2008 with the Max Planck Institute of Quantum Optics (MPQ) in Garching, Germany, which hosts its own attosecond laser, and the Ludwig Maximilian University of Munich.

"It is very exciting that the frontier of attosecond science is now having its outpost in the Gulf state," says Olga Smirnova, an atomic physicist at the Max Born Institute in Berlin.
Saudi Arabia is known for its oil wealth, and in 2002 its government decided that science was the key to incubating a more diverse economy. Its strategy comprises heavy financial investment and forging partnerships with leading research institutions abroad and it seems to be working. In the past five years, the number of scientific papers produced by researchers in Saudi Arabia has skyrocketed. The quality of the research has now overtaken that of Turkey and Iran, according to impact metrics known as SNIP (Source Normalized Impact per Paper) from the University of Leiden in the Netherlands. Prince Turki Bin Saud Bin Mohammad Al Saud, who heads Saudi Arabia's National Science, Technology and Innovation Plan, told Nature's News team that science funding has been doubled from this year and that the country is on track to reach Western levels by the mid-2020s.

Attosecond lasers quickly became fundamental tools in atomic physics after the first attosecond laser pulses were reported in 2001 by a team led by the MPQ's Ferenc Krausz, who heads the Attosecond Science Lab (M. Hentschel et al. Nature 414, 509-513; 2001).

The lasers have since moved into the realm of molecular sciences, including condensedmatter systems and molecular biology, where they are being used to investigate how the movement of electrons can initiate changes in the structure of molecules. "They provide an exquisitely sharp temporal scalpel for dissecting the inner workings of matter," says laser physicist John Tisch of Imperial College London.

One of the first planned experiments for the KSU laser will study the behaviour of electrons in atoms of melanin, best known as the pigment that protects skin from the sun's ultraviolet rays. No one knows why ultraviolet photons do not normally break the chemical bonds in the molecule when they hit it, but it is assumed that melanin's electrons redistribute - and diffuse - the energy among themselves. The experiment at KSU will test this hypothesis by developing extremely short, high-intensity ultraviolet pulses to excite the electrons, and will then capture their movements with the attosecond laser.

The collaboration with Saudi Arabia gives Krausz the chance to enter totally new territory. He will work with oncologist Jean-Marc Nabholtz - who moved to KSU last year to head its National Comprehensive Cancer Center - to adapt the laser to generate 
- pulses of infrared light for analysing proteins and nucleic acids in blood samples from people with cancer. The aim will be to find molecular 'fingerprints' that might diagnose cancers, or predict response to therapy or the future onset of a cancer.

The value of such a source of infrared light, Krausz says, is that a table-topsize laser system could be developed and used at patients' bedsides. Currently, the only sources of such radiation are synchrotrons, which

"They provide an exquisitely sharp temporal scalpelfor dissecting the inner workings of matter." require large, expen-

sive infrastructures. Because Krausz has little experience in this area, it would have been hard for him to obtain funding in Germany for such medical applications, he says.

The place of women in Saudi society and education, and the country's human-rights record, have presented challenges for members of the collaboration. At KSU, which was founded in 1957, male and female students have separate campuses. No rule forbids women from entering the new lab, says Abdallah Azzeer, who leads the KSU side of the laser collaboration, but mixing of the sexes contravenes cultural norms. "We will make special arrangements to ensure their access," he says. One possibility, he adds, might be to train female $\mathrm{PhD}$ students in handling the equipment so that they can supervise female undergraduates whose parents do not want them to attend mixed classes.

Krausz has had to get used to working in a segregated environment during his time at KSU. All the lectures are given in the men's campus and beamed over to the women's campus, and Krausz remembers being extremely startled the first time he received a disembodied question from a female student over loudspeakers.

He thought long and hard about working with Saudi Arabia, he says. As a Hungarian who left for the West in 1987 at the age of 25 , he is hypersensitive to human-rights issues. But not long before he decided to collaborate with KSU in 2008, he had cancelled a trip to China in protest against a clamp-down on press freedom there, and then regretted the decision. It achieved nothing save the embarrassment of the scientists, he says, and he concluded that, in such cases, "the best thing is to talk to each other and learn each other's problems".

He found himself genuinely moved by the enthusiasm for science he encountered on his first visit to KSU later that year. "It felt like a small revolution was happening," he says. "I thought about how I would have felt in the same situation in Hungary - I might have stayed." —

\section{SUMMIT ON CYBERSECURITY AND CONSUMER PROTECTION}

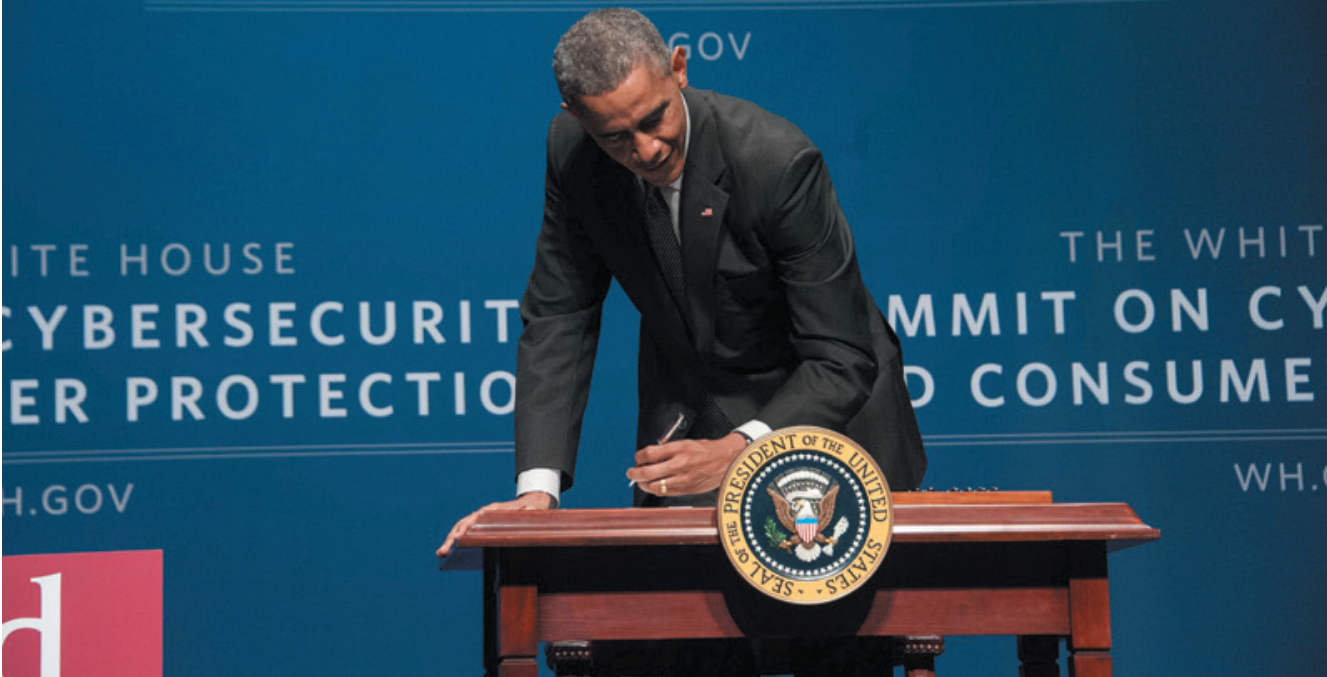

US President Barack Obama has ordered better federal cooperation with private firms to fight hackers.

CYBERSECURITY

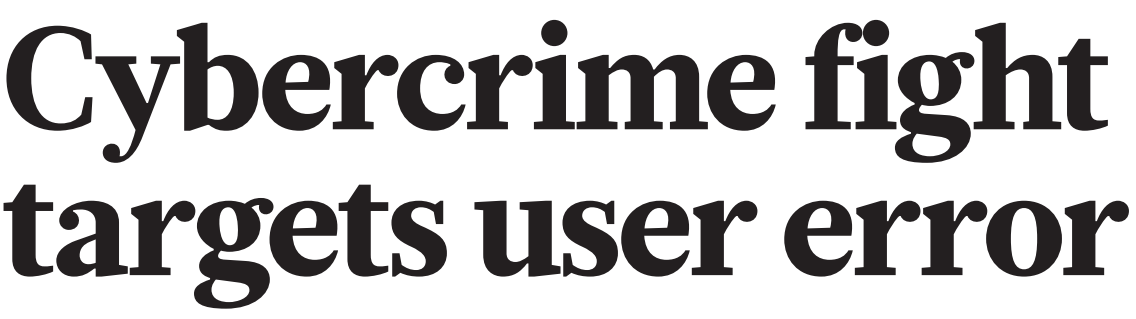

\section{Researchers consider ways to diminish human factors in the equation for keeping data safe.}

\section{BY ERIKA CHECK HAYDEN}

I would be easy to blame the poor soul at Sony Pictures Entertainment who opened the door to one of the most disastrous hacks in history just by clicking an e-mail link. As US President Barack Obama pointed out during a visit to Stanford University in California on 13 February, user negligence is often the key to a successful cyberattack.

"It's just too easy for hackers to figure out usernames and passwords, like 'password'. Or 12345 ... 7," Obama said. But people do this kind of thing all the time, says Angela Sasse, head of information-security research at University College London. Researchers have found that after employees were asked to create long passwords according to strict rules, some of them wrote the password down in an easily accessible place, such as on a desk in plain sight. Other employees might choose to work outside a secured network because it runs too slowly (see also go.nature.com/buxsds).

Such measures confound security experts but are a logical response to the increasing security workload imposed on employees, Sasse says. "We want security that is effective but also allows us to get on with the job," she adds. "A lot of smarter companies are realizing that some of these security measures are a bad productivity drain." Cormac Herley, a security researcher at Microsoft Research in Redmond, $\frac{\text { Tे }}{z}$ Washington, has estimated that the world's Internet users collectively spend the equivalent of 1,389 years every day entering passwords (C. Herley IEEE Secur. Priv. 12, 14-19; 2014).

Generally, the financial services industry is further ahead than others in dealing with the problem, because its business relies on ensuring that customers can easily access their funds while thieves are kept out. But a spectacular failure in its efforts was revealed on 16 February, when the Russian computer-security firm Kaspersky Lab described how hackers had managed to steal an estimated US\$1 billion from financial institutions around the world by infiltrating a bank in Ukraine. As in the Sony case and many others, the fatal security flaw was an errant click on an e-mailed link.

In banking, authentication is the key step 